\title{
Associating liver partition and portal vein ligation for staged hepatectomy: establishment of an animal model with insufficient liver remnant
}

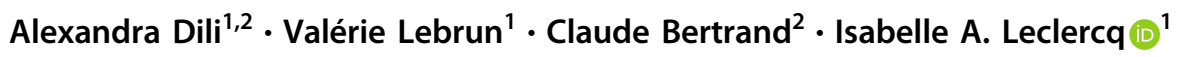

Received: 18 April 2018 / Revised: 14 September 2018 / Accepted: 16 September 2018 / Published online: 21 January 2019

(c) United States \& Canadian Academy of Pathology 2019

\begin{abstract}
Associating liver partition and portal vein ligation for staged hepatectomy (ALPPS) allows extended hepatectomy in patients with an extremely small future liver remnant (FLR). Current rodent models of ALPPS do not include resection resulting in insufficient-for-survival FLR, or they do incorporate liver mass reduction prior to ALPPS. Differences in FLR volume and surgical procedures could bias our understanding of physiological and hemodynamic mechanisms. We aimed to establish a rat ALPPS model with minimal FLR without prior parenchymal resection. In rodents, the left median lobe (LML) represents $10 \%$ of total liver. Partial hepatectomy (PHx) sparing LML and pericaval parenchyma represents our reference $87 \%$ resection. The first step in the procedure is either portal vein ligation (PVL) corresponding to ligation of all but the LML portal branches, or PVL with transection between the left and right median lobe segments (PVLT), and is defined as ALPPS stage-1. Second, ligated lobes were removed: PVL-PHx represents a conventional 2-stage hepatectomy, while PVLT followed by PHx is a strict reproduction of human ALPPS. In Group A, liver hypertrophy was analyzed after PVL $(n=38)$, $\operatorname{PVLT}(n=47), \mathrm{T}(n=10)$, and sham $(n=10)$; In group B, mortality and FLR hypertrophy was assessed after PHx $(n=42)$, Sham-PHx $(n=6)$, PVL-PHx $(n=37)$, and PVLT-PHx $(n=45)$. In group A, PVLT induced rapid FLR hypertrophy compared to PVL $(p<0,05)$. Hepatocyte proliferation was higher in PVLT remnants $(p<0,05)$. In group B, PHx had a 5-day mortality rate of $84 \%$. Sham operation prior to PHx did not improve survival $(p=0.23)$. In both groups, major fatalities occurred within $48 \mathrm{~h}$ after resection. PVL or PVLT prior to PHx reduced mortality to $33.3 \%(p=0,007)$ or $25 \%$ ( $p=$ $0.0002)$ respectively, with no difference between the 2 two-stage procedures $(p=0.6)$. 7-day FLR hypertrophy was higher after the PVLT-PHx compared to PVL-PHx and PHx $(p=0.024)$. Our model reproduces human ALPPS with FLR that is insufficient for survival without liver resection prior to the stage-1 procedure. It offers an appropriate model for analyzing the mechanisms driving survival rescue and increased hypertrophy.
\end{abstract}

\section{Introduction}

Surgical resection is the only treatment with curative intent for patients suffering from a primary or secondary liver tumor. The extend of liver resection depends on tumor location, and/or burden. Case series show that, when

Isabelle A. Leclercq

isabelle.leclercq@uclouvain.be

1 Laboratory of Hepato-Gastroenterology, Institut de Recherche Expérimentale et Clinique,Université catholique de Louvain, Brussels, Belgium

2 Department of Surgery, Centre Hospitalier Universitaire UCLouvain, Brussels, Belgium operated upon, patients with colorectal liver metastases, even multiple and initially unresectable, can achieve higher survival rates compared to chemotherapy alone [1]. Tumor burden but also tumor biology may have an important impact on survival [2,3]. Recent guidelines propose to attempt curative resection $[4,5,6]$, and this is also recommended for multiple metastases in spite of the lack of evidence from randomized controlled trials [7]. In order to propose resection, the surgeon must respect oncological limits and "liver-related" limits determined by the size and function of future liver remnant (FLR). Resection must leave in place at least $20-25 \%$ of the initial liver volume when the parenchyma is healthy, or $40 \%$ in case of diseased liver with reduced functional capacity [8]. If resection is performed without respecting hepatic-related limits, patients risk postoperative liver insufficiency due to 
"small for size syndrome (SFSS)". SFSS is a clinical entity that combines postoperative hyperbilirubinemia, ascites and coagulopathy and is related to high mortality rates $[9,10]$.

To increase, when needed, the size of the future liver remnant, the gold standard is embolization or ligation of portal branches (PVL/E) nourishing the diseased, to-beexcised, liver in order to stimulate hypertrophy of the FLR by compensatory growth [11]. Portal vein occlusion achieves hypertrophy of the future liver remnant up to $52.8 \%$ of its initial size within 4-6 weeks, in some reports. However, up to $30 \%$ of the patients who gain insufficient liver mass recovery or experience a progression of their oncogenic disease during this period will be ineligible for liver resection [12].

Associated liver partition and portal vein ligation for staged hepatectomy (ALPPS) is a technique that combines portal vein ligation and parenchymal in situ split to obtain rapid and enhanced hypertrophy of the future liver remnant [13]. Although the use of the technique is controversial [12], the cautious selection of patients and indications seem paramount to reduce the high morbidity and mortality rates initially reported $[14,15,16]$. ALPPS is proposed for patients with an extremely small FLR [17], or even as a rescue technique for patients whose liver failed to hypertrophy after portal vein occlusion [18]. ALPPS patients obtain accelerated and enhanced FLR hypertrophy [19] without developing liver insufficiency [13, 20], despite the extent of parenchymal resection.

Since the first report published in 2012 [13], experimental attempts have been made to study the mechanism of enhanced FLR hypertrophy in ALPPS. To this end several ALPPS rodent models were developed. However, these models do not include liver resection with insufficient-forsurvival FLR [21-26], or they do incorproate liver mass reduction prior to ALPPS technique [27, 28, 29]. Differences in volume of FLR and in surgical events could introduce bias in our understanding of pathophysiological and hemodynamic mechanisms. This study aimed therefore to establish a rat model of ALPPS with extended hepatectomy $( \pm 90 \%)$ without prior parenchymal resection mimicking the human procedure.

\section{Methods}

\section{Animals}

All animal experiments were conducted in accordance with European regulations and FELASA guidelines for human care for laboratory animals established by the Université catholique de Louvain (UCLouvain, Belgium), and the study protocol was approved by the university ethics committee.
Data were reported according to the ARRIVE guidelines. To avoid gender-related effects only male Wistar rats of $\sim 350 \mathrm{~g}$ (UCLouvain Medical school, Brussels, Belgium) were used. Animals were housed in a temperature- and humiditycontrolled environment in a 12-h light/12-h dark cycle. They had free access to food and water at all times before and after the surgical procedure. In order to take into account circadian variations in liver regeneration, all procedures were performed between 7 and $12 \mathrm{am}$. In a preliminary experiment, we measured the volume of the liver and of each individual lobe in 10 control rats.

\section{Surgical procedures and experimental design}

During the operative procedures, animals were allowed to breathe spontaneously in a glass cylinder filled with a mixture of oxygen (2 1/min) and isoflurane (2.5\%) (IsoFlo, Zoetis BelgiumSA, Louvain-la-Neuve, Belgium). Animals had a median laparotomy after subcutaneous injection of $5 \mathrm{cc}$ of warm physiological sterile solution to increase the circulatory blood volume. Liver ligaments were gently freed and left lateral lobe (LLL) and median lobe (ML) were lifted with cotton-tipped sticks out of the abdomen and wrapped in gauzes to keep them humidified.

Conversely to the human liver, the rat liver is already partitioned, and the only lobe that receives two distinct glissonian pedicles is the median one: the right segment of the median lobe (RML) receives a pedicle directly from the hilum, whereas the left segment (LML) shares a common pedicle with the left lateral lobe (LLL). This bifurcation usually takes place into the LLL parenchyma, rendering the dissection of the portal vein branch from the arterial and biliary elements difficult. Indeed, into the parenchyma, glissonien elements are fragile, adherent, and surrounded by connective tissue. Based on microsurgical techniques already described [30, 31, 32], microscopeassisted dissection ( $\times 20$ magnification) and pedicular hydrodissection permitted to individualize precisely the portal vein branch irrigating the LLL, which is posterior to the arterial and biliary elements. Arterial and biliary elements of the LLL were lifted with a Prolene 7/0 (Ethicon) to avoid damage. Cautious dissection allowed preservation of the whole glissonien pedicle destined to LML. LML represents $10 \%$ of the total rat liver mass $[33,31]$ and can be partitioned from the RML. Therefore, in all procedures (ALPPS, portal vein ligation or extended hepatectomy, see below), LML is the future liver remnant. Another technically difficult part of the operation is the dissection of the right lobe and of the pericaval parenchyma (evaluated at $2-5 \%$ of total liver weight $[33,31]$ ) without damaging the inferior vena cava. Even with gentle fine microdissection, liver pericaval parenchyma could not completely be removed. 


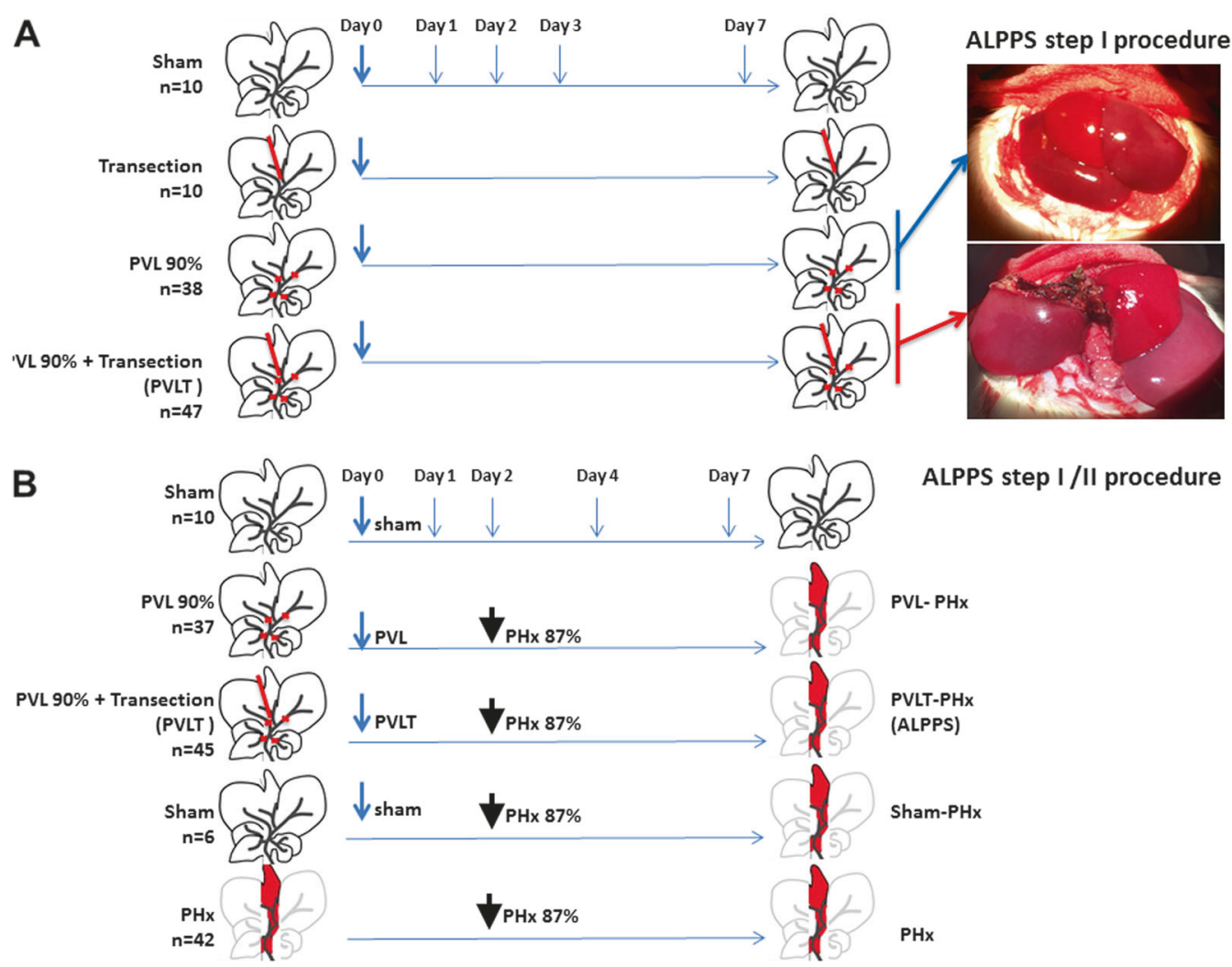

Fig. 1 Schematic representation of the study design for (a) step I in the associating liver partition and portal vein ligation for staged hepatectomy (ALPPS) procedure. Sham $n=10$; Transection: Parenchymal Transection between LMR and left segment of median lobe (LML), $n=5$ per time points; PVL 90\%: Portal vein ligation of all branches but the one irrigating the LML, day $1(n=11)$, day $2(n=5)$, day 3 $(n=5)$, day 7 ( $n=17)$; PVLT: portal vein ligation of all branches but the one irrigating the LML and parenchymal transection between LMR and LML, day $1(n=11)$, day $2(n=7)$, day $3(n=10)$, day 7

\section{Description of the ALPPS rat model}

\section{ALPPS step I (first step procedure): portal vein ligation and transection (PVLT)}

We separately ligated the portal branches for the right, caudate, left lateral lobes and the right segment of the median lobe (90\% PVL). This was combined with complete transection between the left and the right segment of the median lobe (PVLT, $n=47$ ). Rats undergoing PVLT were compared to animals with PVL alone (PVL, $n=38$ ), transection alone (T, $n=10)$ or sham $(n=10)$ (Fig. 1a).

\section{ALPPS step II (complete procedure):}

2 days after PVLT described above, we resected all deportalized lobes leaving in place the LML and pericaval tissue (10 and $3 \%$ of total liver volume, respectively). This 2-step procedure is thereafter denoted as PVLT-PHx or $(n=19)$ and (b) ALPPS step I/step II procedure: PHx: 87\% Partial hepatectomy of all liver segments except the LML and pericaval parenchyma at day 0 , day $1(n=11)$, day $2(n=7)$, day $4(n=5)$, day $7(n=19)$; PVLT-PHx (ALPPS): PVLT at day 0 and resection of all deportalized segments at day 2: day $1(n=11)$, day $2(n=7)$, day 4 $(n=11)$, day $7(n=16)$ after initial operation; Sham: $n=10$. PVLPHx: PVL at day 0 and resection of all deportalized segments at day 2: day $1(n=11)$, day $2(n=5)$, day $4(n=9)$, day $7(n=12)$

ALPPS $(n=45)$. The 2-step procedure undergone by the animals in this group is a strict copy of conventional ALPPS procedure in humans. PVLT-PHx (or ALPPS) was compared to the resection of all deportalized segments 2 days after PVL (PVL-PHx, $n=37$ ), to a one-step extended $87 \%$ hepatectomy (PHx, $n=42$ ) and to an extended hepatectomy 2 days after sham operation (Sham-PHx, $n=6$ ). In all groups partial hepatectomy corresponded to resection of all liver lobes but the LML segment (10\%) and pericaval tissue (3\%) (Fig. 1b).

Each animal was clinically observed during the first $6 \mathrm{~h}$ post-surgery and twice daily thereafter and mortality recorded. At selected time points (1, 2, 4 and 7 days), systemic (cardiac puncture) blood was obtained and liver harvested. When indicated, rats received $50 \mathrm{mg} / \mathrm{Kg} \mathrm{5-}$ bromo-2-deoxyuridine (BrdU) $2 \mathrm{~h}$ prior to harvesting. Our experimental protocol and the number of animals are shown in Fig. 1. In the figure legends, we report the number of sample on which each analysis is performed. 
Fig. $2 \mathrm{LML}$ is the ideal future liver remnant (FLR) as it represents approximatively $10 \%$ of the total liver parenchyma and can be partitioned from its right counterpart Analysis of relative weight of rat liver lobes compared to total liver weight in 10 control rats $(\mathbf{a}+\mathbf{b})$.

Schematic representation of a partial hepatectomy leaving as FLR the left segment of the median and the pericaval tissue ( $87 \%$ of total liver weight) (c). LLL left lateral lobe, LC caudate lobe, $\mathrm{LR}$ right lobe, RML right medial lobe, LML left median lobe, PC pericaval tissue
A
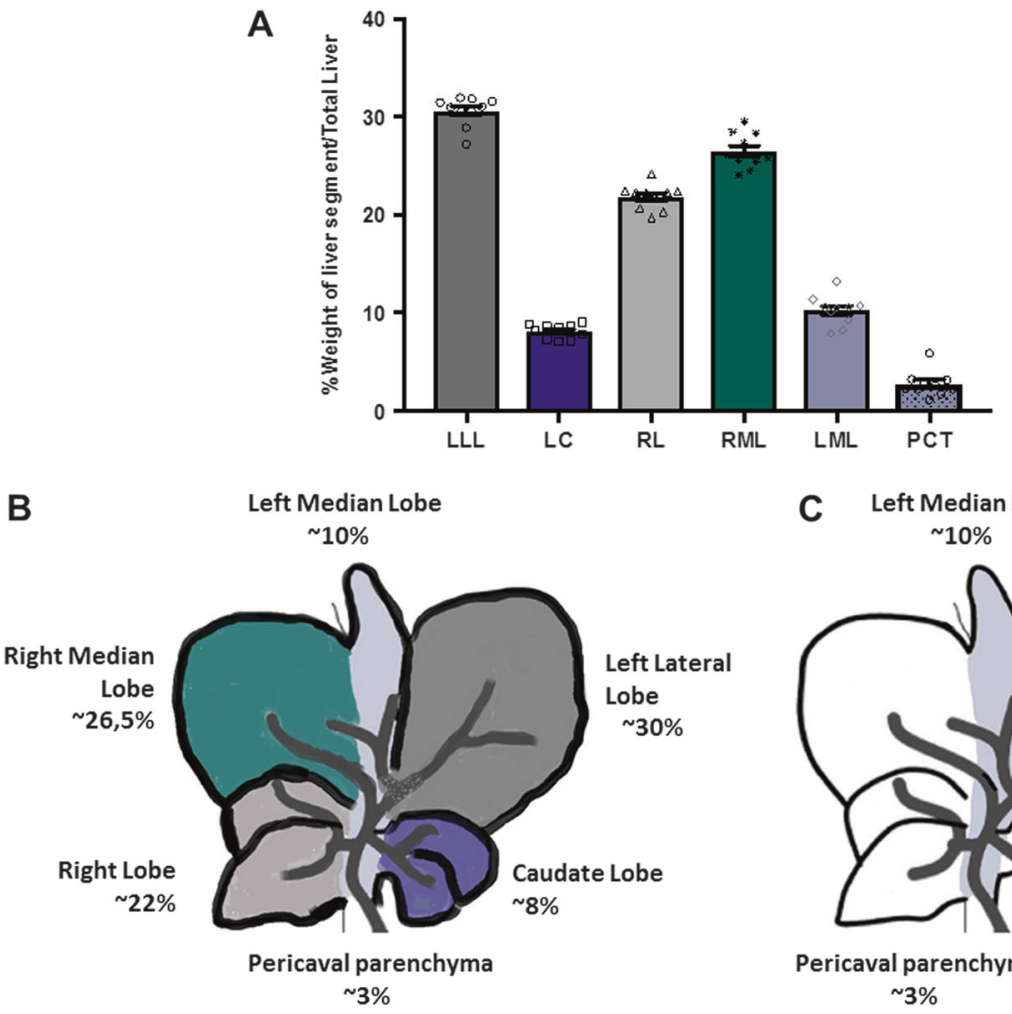

C Left Median Lobe $\sim 10 \%$

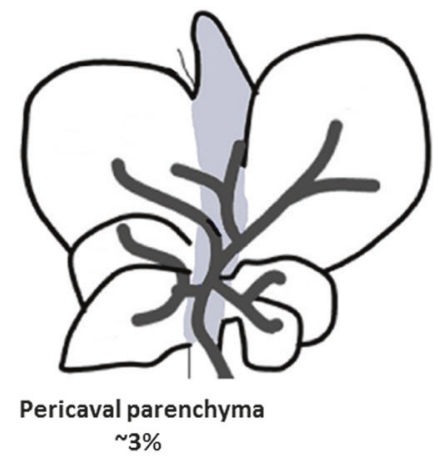

\section{Assessment of liver growth and cell proliferation}

Five markers of liver regeneration were evaluated: (a) The restitution of liver mass was determined as the weight of LML to body weight ratio (FLR/BW); (b) The Kinetic Growth Ratio (KGR) was assessed as the gain of LML weight/BW/day; (c) Immunohistological staining (IHC) and morphometrical quantification of nuclear expression of the proliferation marker Ki67; (d) DNA synthesis determined by immuno-detection of $\mathrm{BrdU}$ incorporated in newly synthesized DNA, and (e) mitotic figures of hepatocytes were counted on 8-10 high power fields ( $\times 20$ magnification) per sample. To confirm the mitotic cell type, we performed double immunofluorescence staining. HNF4 $\alpha$ (R\&D systems; PP-H141500; 1/350) for hepatocyte, pHH3 (Cell Signalling; 9701$\mathrm{S} ; 1 / 200$ ) for mitotic cells, Hoechst for nuclear staining and Lyve-1 (R\&D Systems; AF2125-SP; 1/100) with TSA (Thermo Fisher; Alexa Fluor ${ }^{\mathrm{TM}}$ ) for liver sinusoidal cell.

\section{Liver injury assessment}

Serum aspartate (ASAT) and alanine (ALAT) aminotransferases were measured using an automatic procedure in a serum multiple biochemical analyzer (FUJI DRI-CHEM NX500, Fujifilm Corporation, Tokyo).

\section{Statistical analysis}

GraphPad Prism software (San Diego, CA, USA) was used for graphs and statistics. In graphs, we report individual data (dots), the mean \pm standard error (SEM). Survival curves were analyzed using the LogRang test (Mantel-Cox). Unpaired two-tailed $t$-test was used for simple comparison or one-way and two-way ANOVA followed by Bonferroni's post-hoc correction for multiple comparison. Statistical significance was assumed for $p$ values $<0.05\left({ }^{*} p<0.05\right.$; $* * p<0.01 ; * * * p<0.001)$.

\section{Results}

\section{The left segment of median lobe, an ideal FLR for our study}

Ten rats were harvested as control group to analyze the relative weight of each lobe or segment compared to the total liver weight (Fig. 2a, b). In accordance with other authors [31, 33] the left segment of the median lobe represents $10.24 \%$ (range: $7.29-13.1 \%$ ), while the pericaval parenchyma represents $2.7 \%$ (range: $1.1-5.8 \%$ ) of the total liver (Fig. 2c). Thus, a partial hepatectomy leaving the LML+ pericaval parenchyma as FLR corresponds approximatively to a $87 \%$ hepatectomy. 

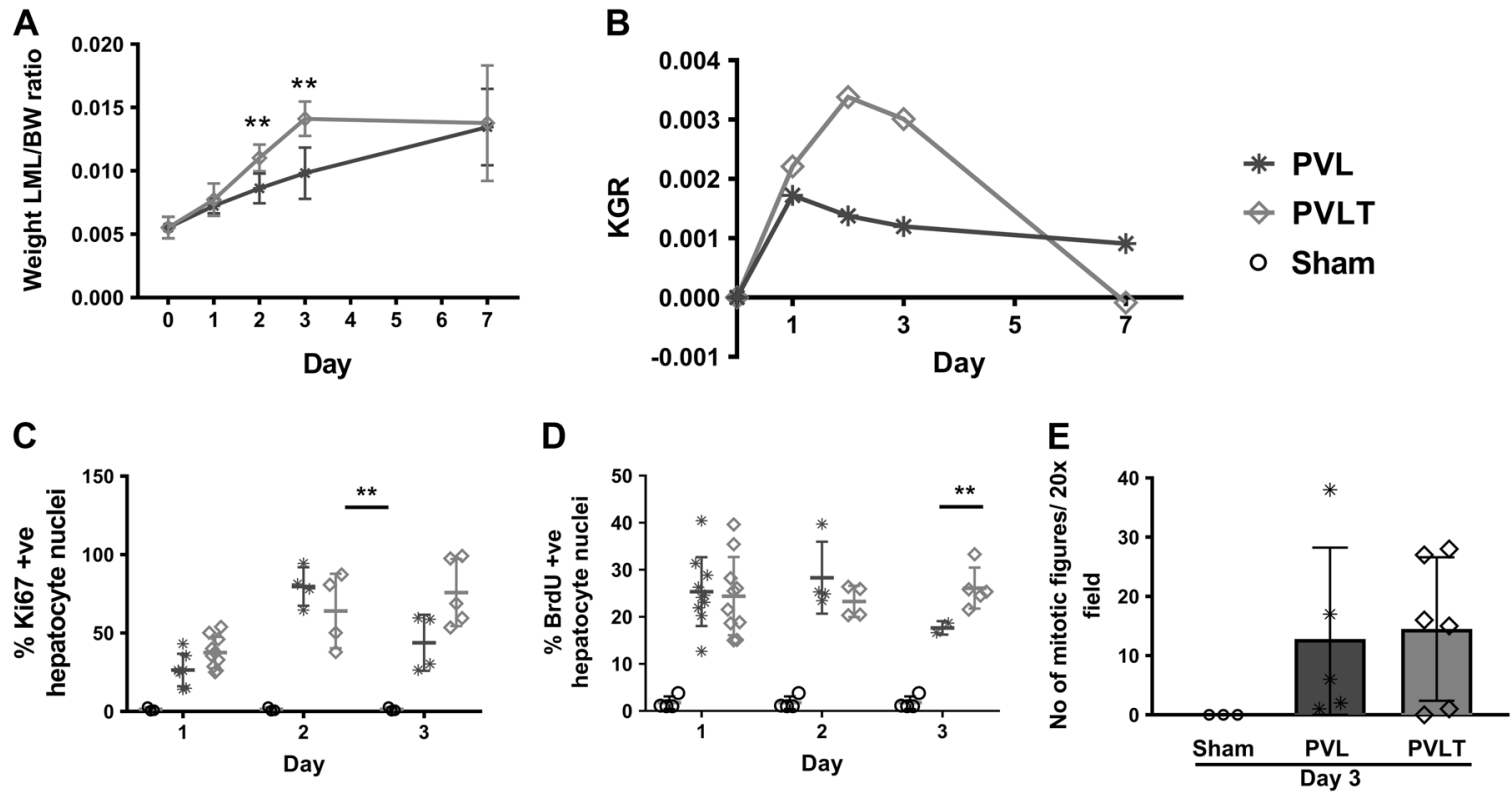

Fig. 3 Accelerated liver regeneration after ALPPS step I procedure. a Weight of future liver remnant (LML)/ body weight evolution (twoway ANOVA: surgery: $p<0.005$, timing: $p<0.0001$; interaction: $\mathrm{ns}$ ), b Kinetic Growth Ratio (KGR) calculated as the gain of LML weight/ BW/day as per Fig. 1a protocol. c Hepatocyte proliferation assessed by quantification of Ki67 positive hepatocyte nuclei after PVLT: day 1 $(n=10)$, day $2(n=4)$, day $3(n=5)$; PVL: day $1(n=7)$, day 2 $(n=4)$, day $3(n=5)$; or Sham: $(n=3)$, (two-way ANOVA: surgery:

\section{Compared to PVL, PVLT (ALPPS step I) boosts the hypertrophy of the FLR}

There was an important release of ASAT and ALAT at day 1 after PVL and PVLT with no significant difference between the groups (data not shown). Both PVL and PVLT induced a hypertrophy of the FLR. The hypertrophy was however greater after PVLT than after PVL at day 2 and 3 $(p<0.05)$ but not at day 7 post-surgery (Fig. 3a). The kinetic growth ratio assessing the daily rate of growth confirmed that the recovery occurred during the first 3 days in PVLT while growth is more constant over the 7 days period after PVL (Fig. 3b). There was no hypertrophy of the LML in animals undergoing transection alone or in shamoperated animals (data not shown). The proportion of cycling hepatocytes, assessed by Ki67 (Figs. 3c, 4c) and the proportion of hepatocytes engaged into the DNA synthesis phase of the cell cycle (BrdU+) (Figs. 3d, 4b) were significantly higher in PVLT than in PVL at day 3. The number of mitotic figures at the 3 day time point was however similar between the 2 groups (Figs. 3f, 4a). Immunohistochemistry and double immunofluorescent staining confirmed that hepatocytes were the main cell type undergoing DNA synthesis and cell division at this time point (Figs. 4 and 5). Thus, PVL associated with $p<0.0001$, timing: $p<0.0001$, interaction: $p<0.001)$. d Quantification of BrdU immunostaining (\% of positive hepatocyte nuclei) after PVLT: day $1(n=10)$, day $2(n=4)$, day $3(n=5)$; PVL: day $1(n=$ $10)$, day $2(n=4)$, day $3(n=2)$, Sham: $(n=4)$, (two-way ANOVA: timing: ns, surgery: $p<0.001$, interaction: ns). e Mitotic count (per $\times 20$ microscopic field) in day $3 \operatorname{PVLT}(n=6)$; PVL $(n=5)$ and sham $(n=3)$ (One-way Anova: ns). $* P<0.05$ and $* * p<0.01$ using Student $t$-test. ns not significant

parenchymal in situ split accelerates FLR hypertrophy compared to PVL without transection.

\section{ALPPS reduces mortality due to SFSS}

Extended $87 \%$ partial hepatectomy (PHx) is associated with a high $84.21 \%$ mortality (16 out of 19) at day 7 (Fig. 6). Sham operation prior to $87 \%$ partial hepatectomy (ShamPHx) did not improve survival compared to the upfront extended hepatectomy $(83.33 \%, 5$ out of $6, p=0.23)$. In both groups, major fatalities occurred within the first $48 \mathrm{~h}$ after extended resection (PHx: 68.4\% and ShamPHx:66.6\%). Thus, the extended PHx provokes a SFSS.

When PHx is preceded by PVL (PVL-PHx) or PVLT (PVLT-PHx or ALPPS) the seven-day mortality caused by the hepatectomy significantly dropped, compared with PHx, to $33.3 \%$ ( 4 out of $12, p=0.007$ ) or $25 \%$ ( 4 out of $16, p=$ 0.0002), respectively, with no significant difference between the 2 two-stage procedures $(p=0.6)$ (Fig. 6a). Our surgical strategy that models ALPPS dramatically reduced the mortality due to SFSS that occurs with a minimal FLR.

5 days after hepatectomy, FLR hypertrophy was significantly higher after the PVLT-PHx (or ALPPS) procedure compared to PVL-PHx $(p=0.024)$ and to SFSS (PHx) survivors $(p=0.024$; Fig. $6 \mathrm{~b})$. 
Fig. 4 Histological illustrations of hepatocyte proliferation PVL and PVLT at day 3 after the operation. Representative pictures from a H\&E-stained liver sections readily showing hepatocyte mitosis (black arrows point to the mitotic figures). $\mathbf{b}$ Identification of BrdU incorporation into newly synthtised DNA. Lower panel are a higher magnification and show BrdU incorporation preferentially in hepatocytes, although few non parenchymal cells (broken-line arrow) are also in the synthesis phase of the cell cycle. c Ki67, a marker of cycling cells, IHC. Bar size: $100 \mu \mathrm{m}$
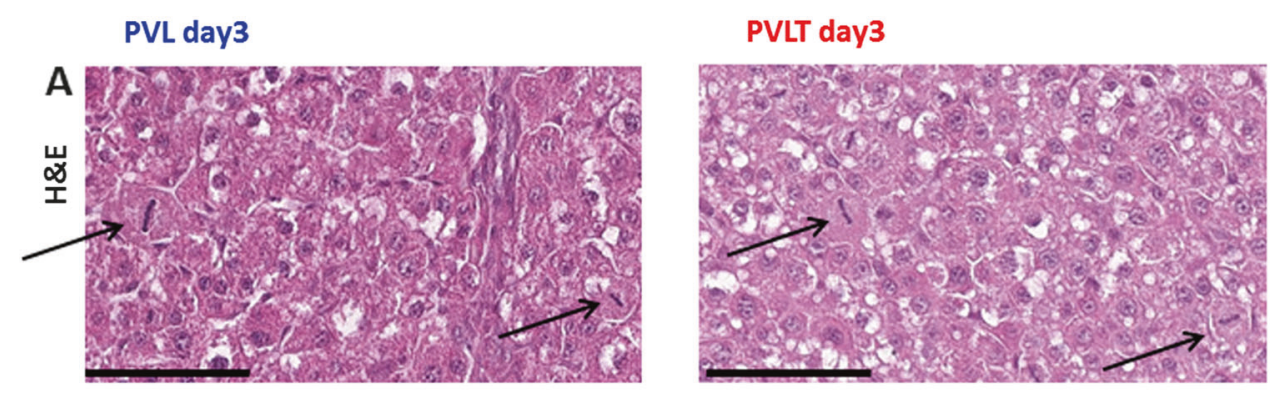

B
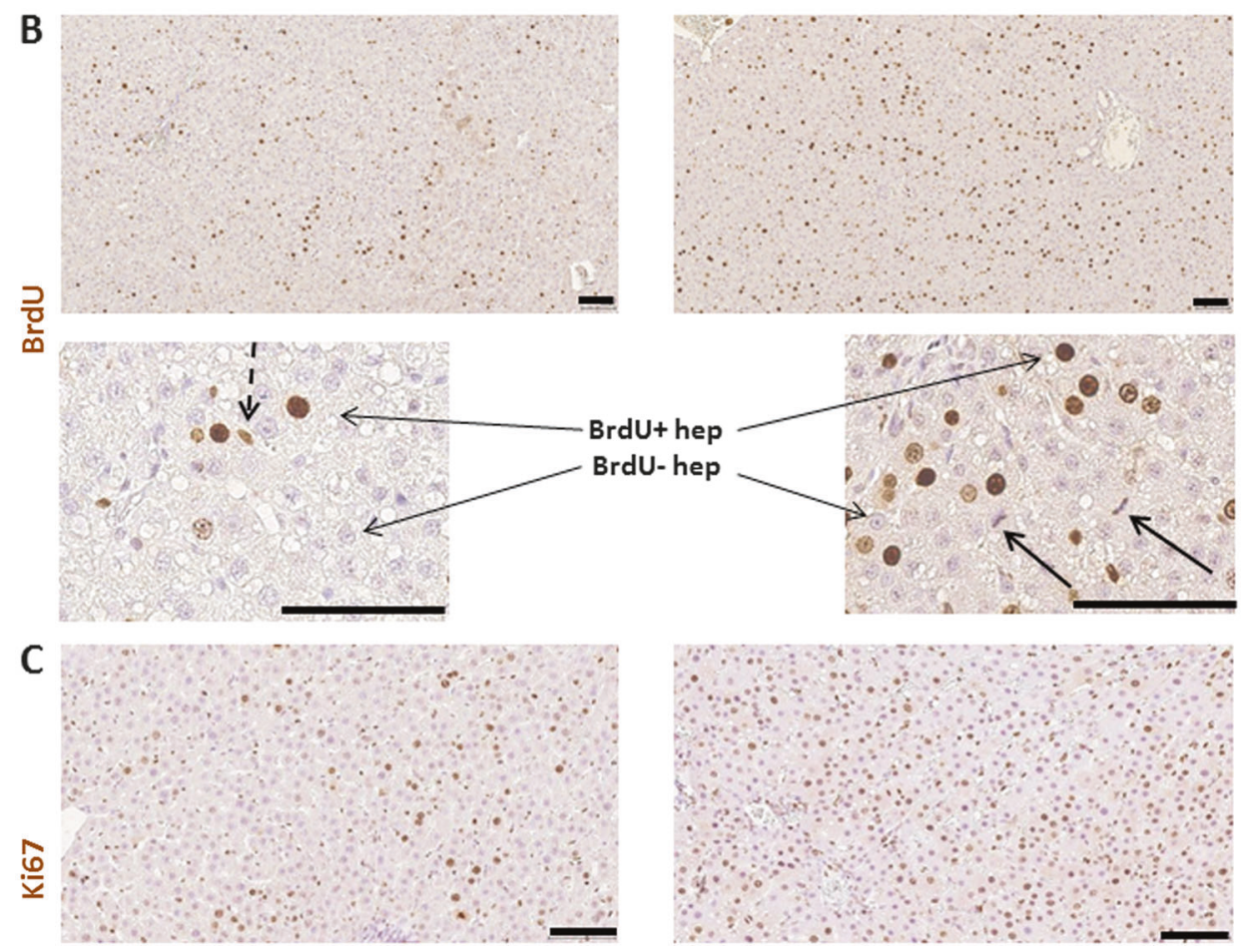

At day 1 after $87 \%$ partial hepatectomy, hepatocyte proliferation was already engaged as shown by the increased number of Ki67 positive hepatocyte nuclei (Fig. 6c). PVL triggered hepatocyte proliferation as at day 1 post PVL the proportion of Ki67 positive nuclei rose to the same extend as after $\mathrm{PHx}(p=0.5)$. Importantly, PVLT alone, on the first day after the initial step, triggered an even higher regenerative response $(p=0.0085$ compared to PHx; Fig. 6c). In addition, at day 5 post hepatectomy (step II procedure), the number of hepatocytes still engaged in DNA synthesis (BrdU + hepatocytes in Fig. 7) or in mitosis was significantly higher in PVLTPHx compared to PVL-PHx ( $p=0.0027$; Fig. 6d). This supports that in addition to initial higher liver hypertrophy obtained after step I procedure, there is a higher rate of hepatocyte proliferation following hepatectomy concurring to increased liver mass recovery after ALPPS (Fig. 6b).

\section{Discussion}

ALPPS is a surgical technique implemented for patients needing hepatectomy with very small FLR. It achieves a rapid boost in FLR hypertrophy compared to the classical two-stage hepatectomy with portal vein ligation/occlusion. This accelerated and enhanced hypertrophy could diminish the drop out of planned resections after portal vein ligation or embolization due to progression of the underlying primary or secondary tumor or insufficient liver regeneration [34].

Since 2014, 9 publications describe rodent models of ALPPS. Dahr et al. initially proposed a model in which the right median lobe (corresponding to $26-28 \%$ of the original liver mass $(+3 \%$ of pericaval liver tissue) is the future liver remnant [21]. This model was subsequently used by several groups [26, 22, 24, 27]. In another model described by Perez et al. [23], the left median lobe, caudate lobe and 
Fig. 5 Immunofluorescent staining of a HFN4a, a hepatocyte specific transcription factor, in red, phophohistone $\mathrm{H} 3$ (PHH3), a marker of mitotic cells, in green and nucleus in blue (DAPI) and b Lyve-1, a endothelial cell marker, in red, $\mathrm{PHH} 3$, in green and nucleus in blue (DAPI) in PVLT liver 3 days after first procedure confirming that hepatocytes (DAPI-HNF $4 \alpha+$ cells) but not LSEC (Lyve-1 + cells) undergo cell proliferation. Bar size $10 \mu \mathrm{m}$
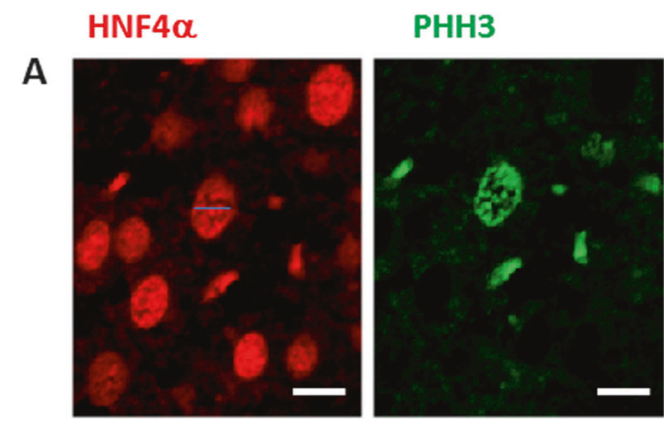

DAPI

HNF4 $\alpha$ PHH3 DAPI

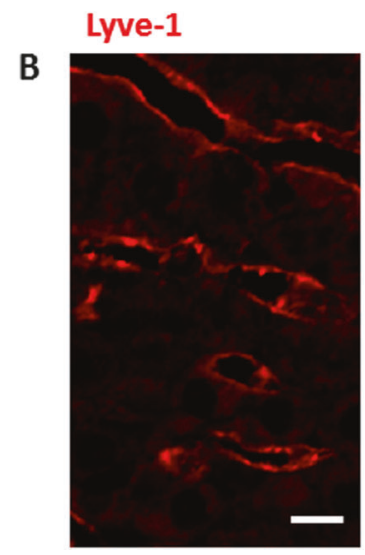

PHH3

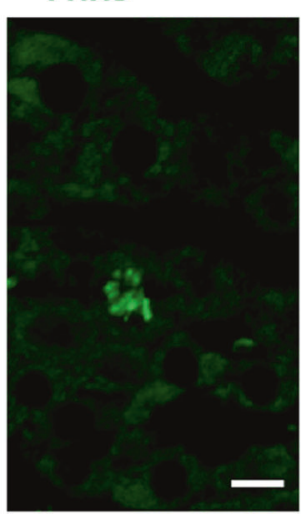

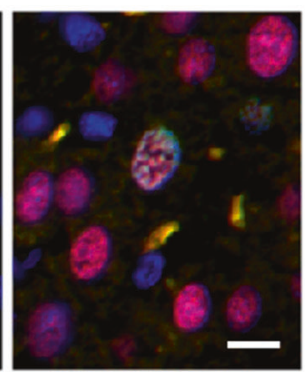

DAPI

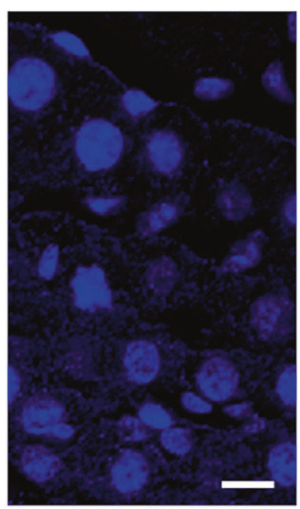

Lyve-1 PHH3 DAPI

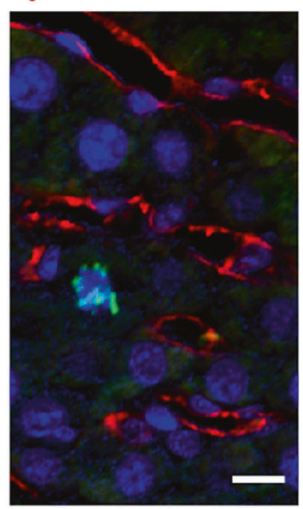

pericaval tissue amounting to $39 \%$ of the total liver volume represent the FLR. It is well documented that the rate of liver regeneration is related to the extent of hepatic resection [35] as well as to the extent of occlusion of the portal bed after PVL [36, 35, 37, 38]. In the context of extended hepatectomy Zhang et al [37] even highlighted that a 5\% difference in the volume resected significantly impacts on mortality and regeneration rate. In all ALPPS models mentioned above, a large future liver remnant is left in place such as the resection corresponds to a $70 \%$ partial hepatectomy, an historical model with excellent survival and in which 7 days suffice to achieve full regeneration [39]. In this regards, the above models do not mimic the minimal, insufficient for survival FLR that would impose an ALPPS procedure in humans.

Other investigators resect part of the liver during the first step of the procedure: Wei and co-workers [27] resected the caudate lobe while Schlegel et al. [28]. and Shi et al. [40]. remove the left lateral lobe in mouse and rat, respectively, at the time of PVLT. This corresponds to an 8-30\% upfront partial hepatectomy respectively or a wedge hepatectomy during human ALPPS procedure. Previous literature abundantly supports that such an hepatectomy cannot be seen as trivial as it is sufficient to prime the liver and engage a regenerative response [41, 42], thereby introducing a confounding factor when analyzing the ALPPS-dependent effects.
In the model we describe here a minimal, incompatible with survival FLR is left in place as proved by the tremendous mortality rate after PHx, unless ALPPS is performed. In addition, no liver tissue is resected during the first step of the procedure. This offers thus a clean model to analyze and understand the mechanisms driving the increase in hypertrophy of the FLR and the rescue of survival. We showed that the first step ALPPS procedure, namely PVL and parenchymal transection, rapidly engaged hepatocytes into cell cycle in an accelerated fashion and to a greater extent that conventional PVL. This accelerated entrance into regeneration allows for significantly increased mass and function recovery and animal survival after hepatectomy.

Metrics in FLR mass recovery were evaluated by mass measurements during harvesting. In order to integrate interindividual differences in animals' body weight, we reported FLR mass as liver mass-to-body weight ratio, a valuable and largely used method to assess liver mass and liver mass recovery. Repeated CT or MRI volumetry, in which each animal serves as its own control, would probably be more adequate to appreciate FLR recovery but this would be at the cost of a higher stress to the animal and perhaps interference with the normal regenerative process. While $90 \%$ partial hepatectomy in rats usually associates with high mortality, some groups report little lethality, especially when the caudate lobe is left in place [31]. The capacity of regeneration of the individual remnant lobes is significantly 

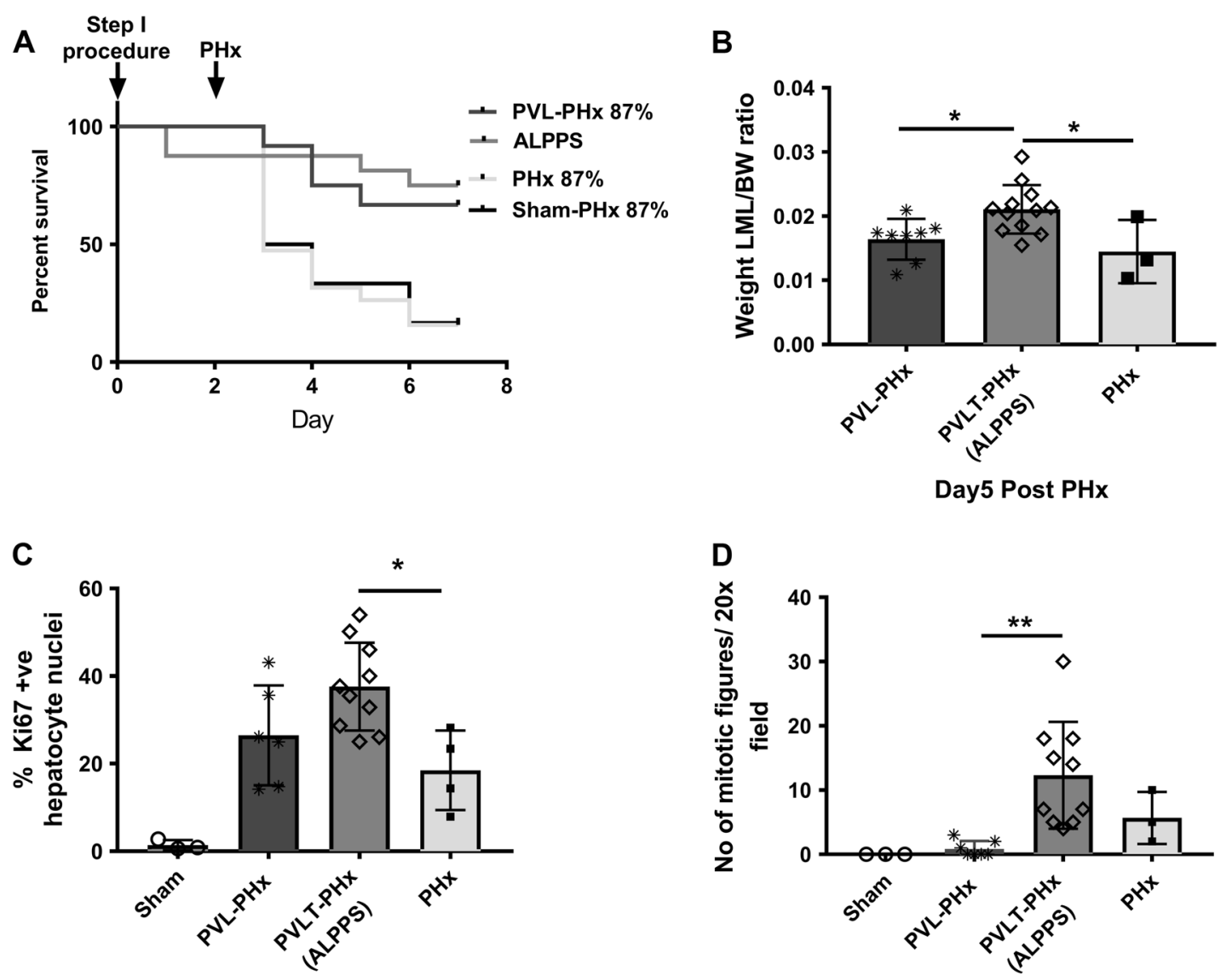

Day 1 post step I procedure

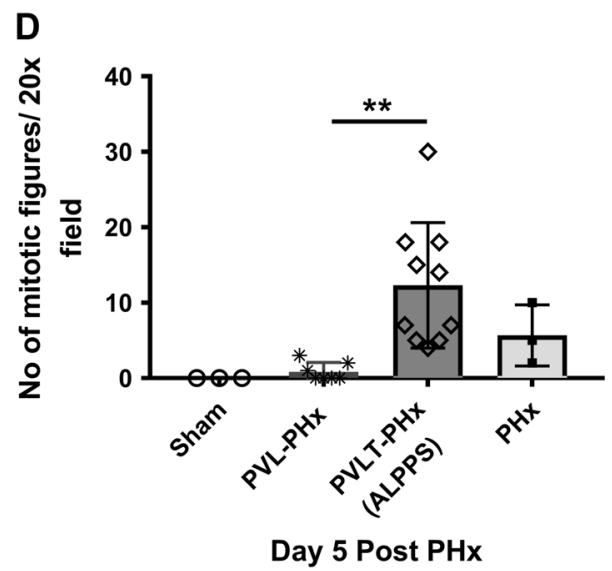

Fig. 6 ALPPS prevents mortality caused by extended hepatectomy. a Kaplan-Meier survival curve after PHx $(n=19)$, PVLT-PHx (or ALPPS) $(n=16)$, PVL-PHx $(n=12)$ and Sham-PHx, $n=6)$. PVLTPHx but also two-stage hepatectomy (PVL-PHx) procedure significantly improved survival ( $p=0.0001$ using Log-rank (MantelCox) test). However, Sham operation prior to $87 \%$ partial hepatectomy had no favorable impact on survival compared to an upfront extended resection ( $p=0.23$ using Log-rank (Mantel-Cox) test). The high mortality rate of PHx proved that our animal model represents SFSS. b Future Liver remnant (FLR) mass recovery after PVLT-PHx procedure compared to extensive $87 \%$ hepatectomy and to PVL-PHx 5 days after surgery (ALPPS $n=12$, PHx $n=3$ survivors, PVL-PHx $n=8)$. One-way ANOVA proved a significant improvement in FLR mass recovery in ALPPS compared to PVL-PHx and to $\mathrm{PHx}(p=$

different [43]. Differences in the nature of the FLR and in surgical dissection imposed by various anatomical localizations, genetic strains of the animals or age at the time of procedure may contribute to variable survival. The experiments reported in this study were performed by a senior surgeon with large experience in rodent liver surgery $(>500)$. In addition, mortality curve in the cohort reported in the paper is similar to that of a separate cohort repeated months later.

In conclusion, we here describe the first rat model with minimal FLR, leading to high mortality due to SFSS unless ALPPS is applied. The degree of liver growth and kinetic growth ratio confirms that in situ split combined to PVL
0.024 , respectively). c Ki67 $\mathrm{HIC}$ at day 1 after operation $(87 \%$ hepatectomy, PVLT $=$ ALPPS step I procedure and PVL $=$ PVL-PHx step I procedure) (Sham $(n=3)$, PVL-PHx $(n=7)$, PVLT-PHx $(n=$ 10), PHx $(n=4))$. ANOVA one-way analysis showed a significant difference in proliferation of PVL-PHx and PVLT-PHx compared to Sham $(p=0.0055$ and $p<0.0001$ respectively). Hepatocyte proliferation was significantly more important in the PVLT-PHx group compared to PHx $(p=0.0085)$, whereas no significant difference was observed between PVL-PHx and PHx operations $(p=0.59)$. d Mitotic count (per $\times 20$ microscopic field) in day 5 post hepatectomy PVLTPHx $(n=10)$; PVL-PHx $(n=7)$, PHx $(n=3)$, and sham $(n=3)$ (Oneway ANOVA and Bonferroni post hoc: $p=0.0027$ in PVLT-PHx compared to PVL-PHx)

boosts liver hypertrophy more than PVL alone. In addition, a second benefice might be expected as hepatectomy induces a higher rate of hepatocyte proliferation that concurs to increased liver mass recovery after ALPPS. This clean model reproduces the objectives intended in human conventional ALPPS and should be of great value for studying the physiological mechanisms leading to accelerated regeneration and rescue from SFSS.

Acknowledgements This project was financially supported by the Fondation Mont-Godinne, Belgium. We thank Dr Caroline Bouzin (I2P, IREC, UCLouvain, Brussels, Belgium), Ms Rita Manco and $\mathrm{Mr}$ Boris Pirlot (GAEN Lab, IREC, UCLouvain, Brussels, Belgium) for technical assistance, Prof. Yves Horsmans (St Luc university Hospital, 

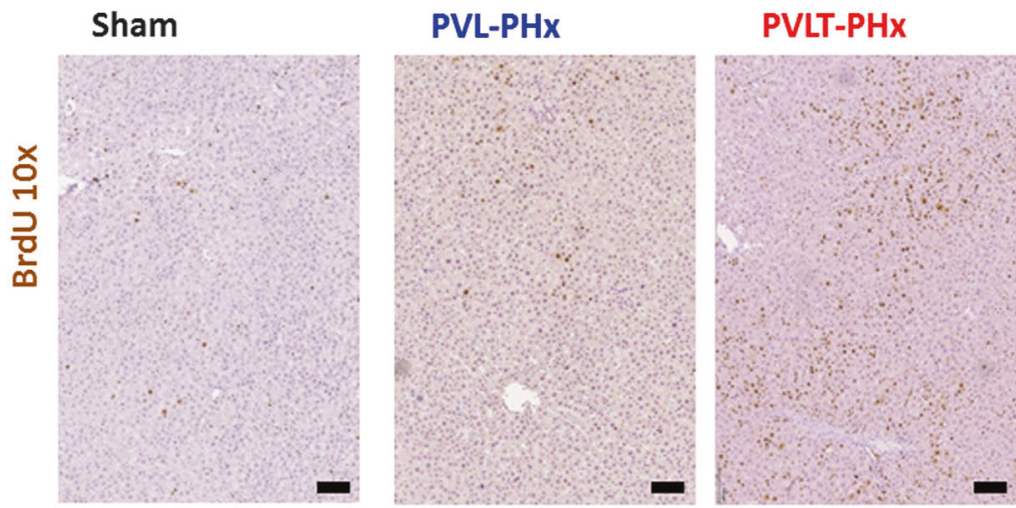

\section{PHx}
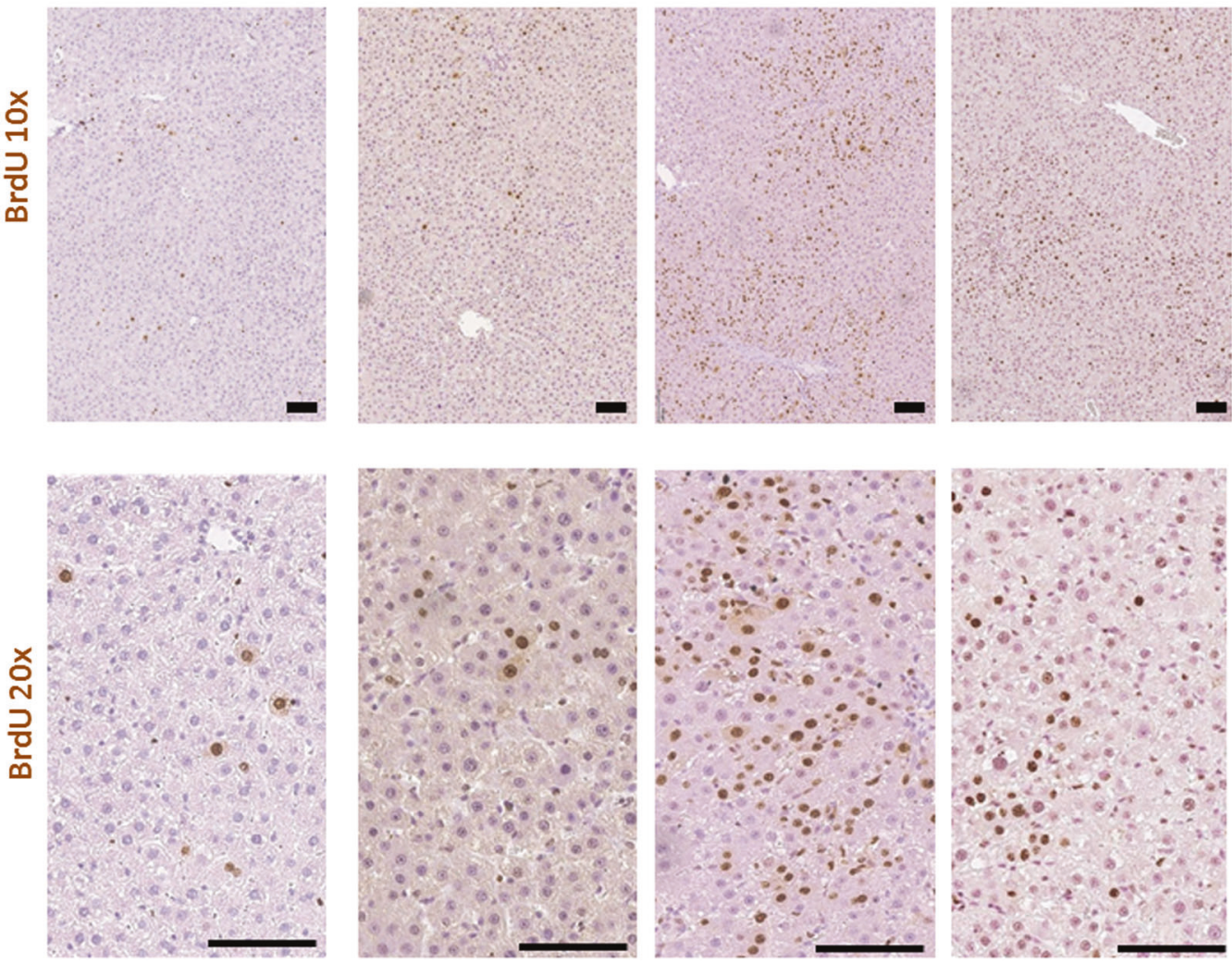

Fig. $7 \mathrm{BrdU}$ immunostaining in Sham, PVL-PHx, PVLT-PHx

(upper panels) and higher magnification (lower panels). Bar size: (ALPPS), PHx 5 days post hepatectomy at low magnification $100 \mu \mathrm{m}$

UCLouvain, Brussesls, Belgium) and Dr Carola Dahrenmöller (GAEN Lab, IREC, UCLouvain, Brussels, Belgium) for critical review of the manuscript.

\section{Compliance with ethical standards}

Conflict of interest The authors declare that they have no conflict of interest.

Publisher's note: Springer Nature remains neutral with regard to jurisdictional claims in published maps and institutional affiliations.

\section{References}

1. Brouquet A, Abdalla EK, Kopetz S, et al. High survival rate after two-stage resection of advanced colorectal liver metastases: response-based selection and complete resection define outcome. J Clin Oncol. 2011;29:1083-90.

2. Blazer DG, Kishi Y, Maru DM, et al. Pathologic response to preoperative chemotherapy: a new outcome end point after resection of hepatic colorectal metastases. J Clin Oncol. 2008;26:5344-51.

3. Sasaki K, Morioka D, Conci S, et al. The tumor burden score: a new "metro-ticket" Prognostic Tool for colorectal liver metastases based on tumor size and number of tumors. Ann Surg. 2018;267:132-41.

4. Garden OJ, Rees M, Poston GJ, et al. Guidelines for resection of colorectal cancer liver metastases. Gut. 2006;55:1-8.
5. Adams RB, Aloia TA, Loyer E, Pawlik TM, et al. Selection for hepatic resection of colorectal liver metastases: expert consensus statement. HPB. 2013;15:91-103.

6. Charnsangavej C, Clary B, Fong Y, Grothey A, et al. Selection of patients for resection of hepatic colorectal metastases: expert consensus statement. Ann Surg Oncol. 2006;13:1261-8.

7. Veereman G, Robays J, Verleye L, et al. Pooled analysis of the surgical treatment for colorectal cancer liver metastases. Crit Rev Oncol Hematol. 2015;94:122-35.

8. Pruvot FR, Truant S. Major hepatic resection: from volumetry to liver scintigraphy. HPB. 2016;18:707-8.

9. Dahm F, Georgiev P, Clavien PA, et al. Small-for-size syndrome after partial liver transplantation: definition, mechanisms of disease and clinical implications. Am J Transplant. 2005;5:2605-10.

10. Asencio JM, García Sabrido JL, Olmedilla L. How to expand the safe limits in hepatic resections? J Hepatobiliary Pancreat Sci. 2014;21:399-404.

11. Makuuchi M, Thai BL, Takayasu K, et al. Preoperative portal embolization to increase safety of major hepatectomy for hilar bile duct carcinoma: a preliminary report. Surgery. 1990;107: $521-7$.

12. Sun Z, Tang W, Sakamoto Y, et al. A systematic review and metaanalysis of feasibility, safety and efficacy of associating liver partition and portal vein ligation for staged hepatectomy (ALPPS) versus two-stage hepatectomy (TSH). Biosci Trends. 2015;9:284-8.

13. Schnitzbauer AA, Lang SA, Goessmann $\mathrm{H}$, et al. Right portal vein ligation combined with in situ splitting induces rapid left lateral liver lobe hypertrophy enabling 2-staged extended right hepatic resection in small-for-size settings. Ann Surg. 2012;255:405-14. 
14. Schadde E, Schnitzbauer AA, Tschuor C, et al. Systematic review and meta-analysis of feasibility, safety, and efficacy of a novel procedure: associating liver partition and portal vein ligation for staged hepatectomy. Ann Surg Oncol. 2015;22:3109-20.

15. Schadde E, Ardiles V, Robles-Campos R, et al. Early survival and safety of ALPPS. Ann Surg. 2014;260:829-38.

16. Rosok B, Bjrnsson B, Sparrelid E, et al. Scandinavian multicenter study on the safety and feasibility of the associating liver partition and portal vein ligation for staged hepatectomy procedure. Surgery. 2016;159:1279-86.

17. Schadde E, Malagó M, Hernandez-Alejandro R, et al. Monosegment ALPPS hepatectomy: extending resectability by rapid hypertrophy. Surgery. 2015;157:676-89.

18. Tschuor C, Croome KP, Sergeant G, et al. Salvage parenchymal liver transection for patients with insufficient volume increase after portal vein occlusion - an extension of the ALPPS approach. Eur J Surg Oncol. 2013;39:1230-5.

19. Montalvá Orón EM, Maupoey Ibáñez J, Bañuelos Carrillo R, et al. Monosegment ALPPS: a new variant of the techniques for rapid hepatic regeneration. Critical review of the initial results of our series. Cir Esp. 2016;93:436-43.

20. Linecker M, Björnsson B, Stavrou GA, et al. Risk adjustment in ALPPS is associated with a dramatic decrease in early mortality and morbidity. Ann Surg. 2017;266:779-86.

21. Dhar DK, Mohammad GH, Vyas, S et al. A novel rat model of liver regeneration: possible role of cytokine induced neutrophil chemoattractant - 1 in augmented liver regeneration. Ann Surg Innov Res. 2015;9:1-10.

22. Almau Trenard HM, Moulin LE, Padín JM, Stringa P, Gondolesi GE, Barros Schelotto P. Development of an experimental model of portal vein ligation associated with parenchymaltransection (ALPPS) in rats. Cir Esp. 2014;92:676-81. https://doi.org/10. 1016/j.ciresp.2013.11.005

23. García-Pérez R, Revilla-Nuin B, Martínez CM, et al. Associated Liver Partition and Portal Vein Ligation (ALPPS) vs selective Portal Vein Ligation (PVL) for staged hepatectomy in a rat model. Similar regenerative response? PLoS ONE. 2015;10:1-14.

24. Schadde E, Tsatsaris C, Swiderska-Syn M, et al. Hypoxia of the growing liver accelerates regeneration. Surgery. 2017;161:666-79.

25. Budai A, Fulop A, Hahn O, et al. Animal models for associating liver partition and portal vein ligation for staged hepatectomy (ALPPS): achievements and future perspectives. Eur Surg Res. 2017;58:140-57.
26. Yao L, Li C, Ge X, et al. Establishment of a rat model of portal vein ligation combined with in situ splitting. PLoS ONE. 2014;9:1-8.

27. Wei W, Zhang T, Zafarnia S, et al. Establishment of a rat model: Associating liver partition with portal vein ligation for staged hepatectomy. Surgery. 2016;159:1299-307.

28. Schlegel A, Lesurtel M, Melloul E, et al. ALPPS: from humain to mice highlighting accelerated and novel mechanisms of liver regeneration. Ann Surg. 2014;260:839-47.

29. Shi H, Yang G, Zheng T, et al. A preliminary study of ALPPS procedure in a rat model. Sci Rep. 2015;5:17567.

30. Aller MA, Arias N, Prieto I, et al. A half century (1961-2011) of applying microsurgery to experimental liver research. World $\mathrm{J}$ Hepatol. 2012;4:199-208.

31. Madrahimov N, Dirsch O, Broelsch C, et al. Marginal hepatectomy in the rat. Ann Surg. 2006;244:89-98.

32. Martins PauloNey, Aguiar PN. Hepatic lobectomy and segmentectomy models using microsurgical techniques. Microsurgery. 2008;28:187-91.

33. Martins PNA, Theruvath TP, Neuhaus P. Rodent models of partial hepatectomies. Liver Int. 2008;28:3-11.

34. Van Lienden KP, Van Den Esschert JW, De Graaf W, et al. Portal vein embolization before liver resection: a systematic review. Cardiovasc Intervent Radiol. 2013;36:25-34.

35. Meier M, Andersen KJ, Knudsen AR, et al. Liver regeneration is dependent on the extent of hepatectomy. J Surg Res. 2016;205:76-84.

36. Lauber DT, Tihanyi DK, Czigany Z, et al. Liver regeneration after different degrees of portal vein ligation. J Surg Res. 2016;203:451-8.

37. Zhang DX, Li CH, Zhang AQ, et al. mTOR-dependent suppression of remnant liver regeneration in liver failure after massive liver resection in rats. Dig Dis Sci. 2015;60:2718-29.

38. Rozka J, Jeppsson B, Bengmark S. Portal branch ligation in the rat reevaluation of a model. Am J Pathol. 1986;125:300-8.

39. Mao SA, Glorioso JM, Nyberg SL. Liver regeneration. Transl Res. 2014;163:352-62.

40. Shi H, Yang G, Zheng T, et al. A preliminary study of ALPPS procedure in a rat model. Sci Rep. 2015;5:1-12.

41. Michalopoulos GK. Liver regeneration after partial hepatectomy. Am J Pathol. 2010;176:2-13.

42. Fausto N. Liver regeneration. J Hepatol. 2000;32:19-31.

43. Inderbitzin D, Studer P, Sidler D, et al. Regenerative capacity of individual liver lobes in the microsurgical mouse model. Microsurgery. 2006;26:465-9. 